\title{
Cone beam CT-based measurement of the accessory mental foramina in the Chinese Han population
}

\author{
LEI XIAO ${ }^{1 *}$, WENJING PANG $^{2 *}$, HONGGUANG BI $^{1 *}$ and XUELIAN HAN ${ }^{3}$ \\ ${ }^{1}$ Department of Stomatology, The Affiliated Yantai Yuhuangding Hospital of Qingdao University; \\ ${ }^{2}$ Yantai No. 1 Middle School of Shandong, Yantai, Shandong 264000; ${ }^{3}$ Department of Stomatology, \\ Traditional Chinese Medicine Hospital of Rizhao, Rizhao, Shandong 276800, P.R. China
}

Received June 13, 2019; Accepted February 5, 2020

DOI: $10.3892 /$ etm.2020.8954

\begin{abstract}
Anatomical data of accessory mental foramina (AMFs) were investigated in a Chinese Han population using cone beam CT (CBCT). A retrospective analysis was performed on 527 selected sets of CBCT images. The average frequency and diameter of AMFs, the diameter of the ipsilateral mental foramen (MF), and the center distance and relative position between the AMFs and MF were measured and calculated by three professional dentists. Among the 527 patients, AMFs were identified in 36 cases (frequency $6.83 \%$ ), of which $68.75 \%$ of AMFs were larger than $1 \mathrm{~mm}$. The mean diameters of the AMFs and the ipsilateral MF were $1.32 \pm 0.61 \mathrm{~mm}$ and $3.26 \pm 0.90 \mathrm{~mm}$, respectively. The average distance from the AMFs to the alveolar ridge crest (ARC) was $15.05 \pm 3.50 \mathrm{~mm}$, and the average distance to the mandibular plane was $15.87 \pm 3.64 \mathrm{~mm}$. The positions of the AMFs relative to the MF varied widely. The AMFs were mostly positioned distal-inferior to the ipsilateral MF and under the mandibular second premolars. Nutrient foramina around the MFs were distinguished from AMFs. The reference plane for measuring AMFs was suggested to be the mandibular plane to increase the repeatability and accuracy of the experiment. Standard planes were proposed to determine the relative position between AMFs and the MFs. Based on our results, we propose that for implant surgeries, the safety region of $2 \mathrm{~mm}$ above the MFs should be reevaluated. CBCT examination is recommended before the operation to identify important anatomical structures around the MF region and their variations and set the safety distance on an individual basis.
\end{abstract}

Correspondence to: Dr Xuelian Han, Department of Stomatology, Traditional Chinese Medicine Hospital of Rizhao, 35 Wanghai Road, Rizhao, Shandong 276800, P.R. China

E-mail: emaf97@163.com

${ }^{*}$ Contributed equally

Key words: accessary mental foramen, mental foramen, cone-beam CT

\section{Introduction}

With the increased demand for dental implantation, mental cosmetic surgery and orthognathic surgery, the study of the anatomical structure of the mandible is receiving increasing attention. The mandibular canal is an important anatomical structure in the mandible, and its course follows a specific path. The mental canal and the incisor nerve canal are separated from the course of the mandibular canal at the premolar region. The mental canal forms two backward, upward and outward foramina, which are called the mental foramina (MFs), usually on each side of the mandible (1). However, some studies have found the presence of one or more accessory mental foramina (AMFs), which are buccal foramina formed by branches of the mental canal (2). The accessory mental vascular bundles transmitted from the AMFs are distributed in the skin and mucous membrane from the mouth to the middle of the lower lip on the same side, innervating the mucous membrane and the skin of the corner of the mouth and the cheek (3). These anatomical structures are associated with rare and previously unexplained complications related to implant surgery and failure. Therefore, understanding the anatomical features of the AMFs is beneficial to avoid injury of the submental nerve and is of great significance for mandibular implant and alveolar surgery. Currently, there is no large-scale study on the anatomical characteristics of AMFs in the Chinese Han population, and research on AMFs has yet to reach a unified conclusion. Therefore, in this study, cone beam computed tomography (CBCT) was used to investigate the anatomical data of AMFs in the Chinese Han population to provide information for clinical implant surgery at the MF region and chin surgery.

\section{Patients and methods}

Patients. This study enrolled 527 patients who received diagnostic or therapeutic CBCT at the Yantai Yuhuangding Hospital between January and May 2017, including 256 males and 271 females, aged between 7-88 years (age distribution shown in Table I). The inclusion criteria were as follows: Chinese Han ethnicity; no apical lesion near the mandibular MF; no history of mandibular fracture or orthognathic 
surgery; no pathological damage to the mandible, including cysts, tumors and unerupted teeth. The exclusion criteria were as follows: presence of mandibular nutrient foramina, and/or discontinuous buccal foramina of the mandibular nerve canal; unclear image at the MF region caused by permanent plaque; and poor CBCT image quality caused by various factors, e.g., patient movement, metal artifacts and operation error.

The study was approved by the Ethics Committee of The Affiliated Yantai Yuhuangding Hospital of Qingdao University (Yantai, China). Signed informed consent was obtained from the patients and/or their guardians.

Equipment and software. All original CBCT volume data images were collected by professional radiologists on a Planmeca ProMax 3D Max CBCT machine (Finland) set at $90 \mathrm{kV}, 8 \mathrm{~mA}, 200$ volume pixels and a $110 \mathrm{~mm} \times 130 \mathrm{~mm}$ field of vision (FOV). Before scanning, all images were set at a size of $501 \times 501 \times 501$. After the examination, all scanned images were transmitted to Lenovo M4650-N000 computers with 1600x900 pixel monitor resolution for 3D reconstruction and analysis with the built-in CBCT software Planmeca Romexis 3.8.3.R.

\section{Measurement of AMF}

Establishment of the reference plane. Given the potential differences in the fixed head positions of patients during CBCT examination, there could be forward/rearward tilt, sideward tilt and rotation in the CBCT images; therefore, the original volume data images needed to be processed before measurement and analysis. The horizontal plane standard of the volume data images was adjusted to parallel to each patient's mandibular plane. The reference plane, which was formed by the lines connecting the menton (Me) and bilateral gonions (Go) (Fig. 1), was used to adjust the sagittal, coronal and axial planes of the volume data images to correct possible oblique head positions of the patients during image acquisition. The interval between data layers was set to $0.2 \mathrm{~mm}$. The grayscale level, contrast and sharpness of the image were adjusted to create the desired data images, and the range of measurements was determined.

Data measurement. The relatively clear positions of the MFs, AMFs and adjacent structures on the 3D reconstruction model were used as references to make fine adjustments on the 2D CBCT images, confirm AMFs, exclude nutrient foramina, identify the layer (Fig. 2) in which the MFs and AMFs were the most clear on the mandibular surface and finally capture this CBCT image to measure of the following data (Fig. 3): i) the diameters of the AMFs and the ipsilateral MF, which evaluated the importance of the neurovascular bundle in the AMFs; ii) the distances between the centers of the AMFs and the ipsilateral MF, from the centers of the AMFs to the alveolar ridge crest (ARC), and from the centers of the AMFs to the mandibular plane, which were used to confirm the relative position of the AMFs in the mandible; iii) the positional relationship between the AMFs and the ipsilateral MF: the tangent of the axial plane, which was parallel to the mandibular plane and passed through the MFs, on the buccal cortical surface of the mandible was used as the horizontal
Table I. Age distribution of the patients.

\begin{tabular}{lr}
\hline Age group (years) & $\mathrm{N}$ \\
\hline $7-18$ & 85 \\
$18-40$ & 167 \\
$40-60$ & 201 \\
$61-88$ & 74 \\
\hline
\end{tabular}

axis. The tangent of the coronal plane, which was vertical to the mandibular plane and passed through the MFs, on the buccal cortical surface of the mandible was used as the vertical axis. The two axes accurately divided the MF region into four quadrants: the mesial-superior, mesial-inferior, distal-superior and distal-inferior regions (Fig. 4). These parameters established a new partitioning for the MFs to precisely distinguish the positional relationship between the AMFs and the ipsilateral MF; iv) the positional relationship between the AMFs and adjacent teeth: below the first premolar, between the first and second premolar, below the second premolar, between the second premolar and the first molar, and below or behind the first molar. These indexes were used to determine the relative positions between the AMFs and adjacent teeth. All image measurements, data records and statistical analyses were performed by three experienced professional dentists, and average values were calculated.

Statistical analysis. Statistical analysis was performed using SPSS 23.0 software (IBM Corp.). Measurement data are shown as the means \pm standard deviations (SDs). Comparison between left and right was performed using a paired t-test. Correlations between the diameters of the AMFs and the ipsilateral MF and among the diameters of the AMFs, the ipsilateral MF and other lengths were analyzed by Pearson's correlation. Differences with $\mathrm{P}<0.05$ were considered statistically significant.

\section{Results}

Measurement repeatability. There were 3 observers involved in this study. They measured the diameters of the AMFs and the ipsilateral MF and the distance between the centers of the AMFs and the ipsilateral MF. The repeatability of the measurement of the incidence of AMFs and of the relative position between the AMFs and the ipsilateral MF or adjacent teeth was $100 \%$.

Incidence of $A M F$. Then the incidence of AMFs among patients was evaluated. The results showed (Table II) that the incidence of AMFs was $6.83 \%$ (36 out of 527 patients), with 18 cases in males $(3.42 \%)$ and 18 cases in females $(3.42 \%)$. There was no significant difference in AMF incidence between sexes ( $\mathrm{P}>0.05)$. AMFs occurred unilaterally in 31 cases $(5.88 \%)$ and bilaterally in 5 cases $(0.95 \%)$. Among the patients identified with AMFs, the majority (28 patients, $77.78 \%$ ) had a single AMF. For the rest, there were 2 cases $(5.56 \%)$ with 2 AMFs on one side, 1 case (2.78\%) with 4 AMFs on one side (Fig. 5), 4 cases $(11.11 \%)$ with 1 AMFs on each side and 1 case $(2.78 \%)$ 

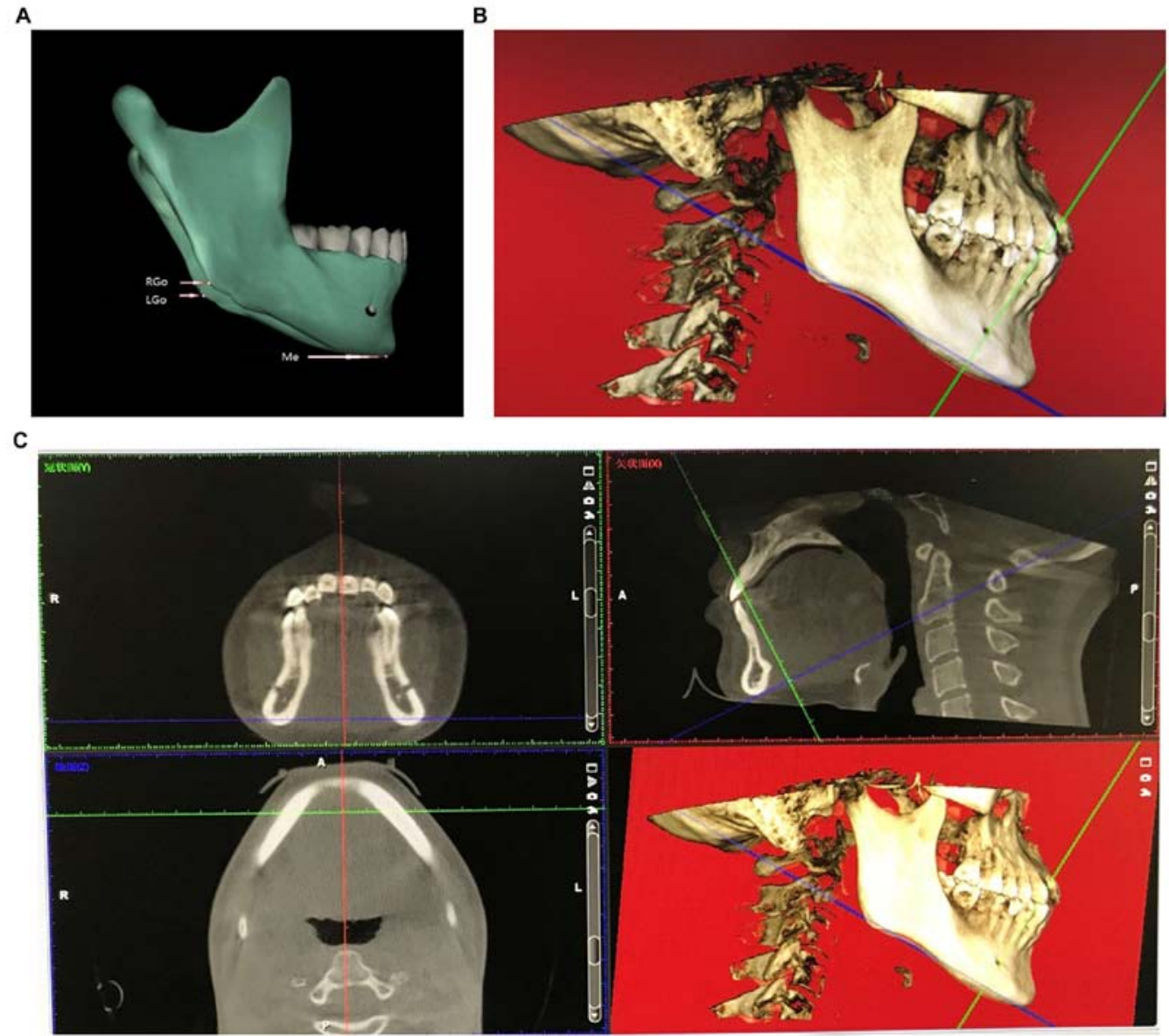

Figure 1. Adjustment of the reference plane. (A) Arrows mark the menton (Me), right gonion (RGo) and left gonion (LGo). (B) The Me and Go were adjusted at the axial layer plane, and the cutting line of the axial plane was adjusted to form a reference plane using the Me and two Go. (C) The sagittal, coronal and axial plane after adjustment and the 3D reconstruction image.
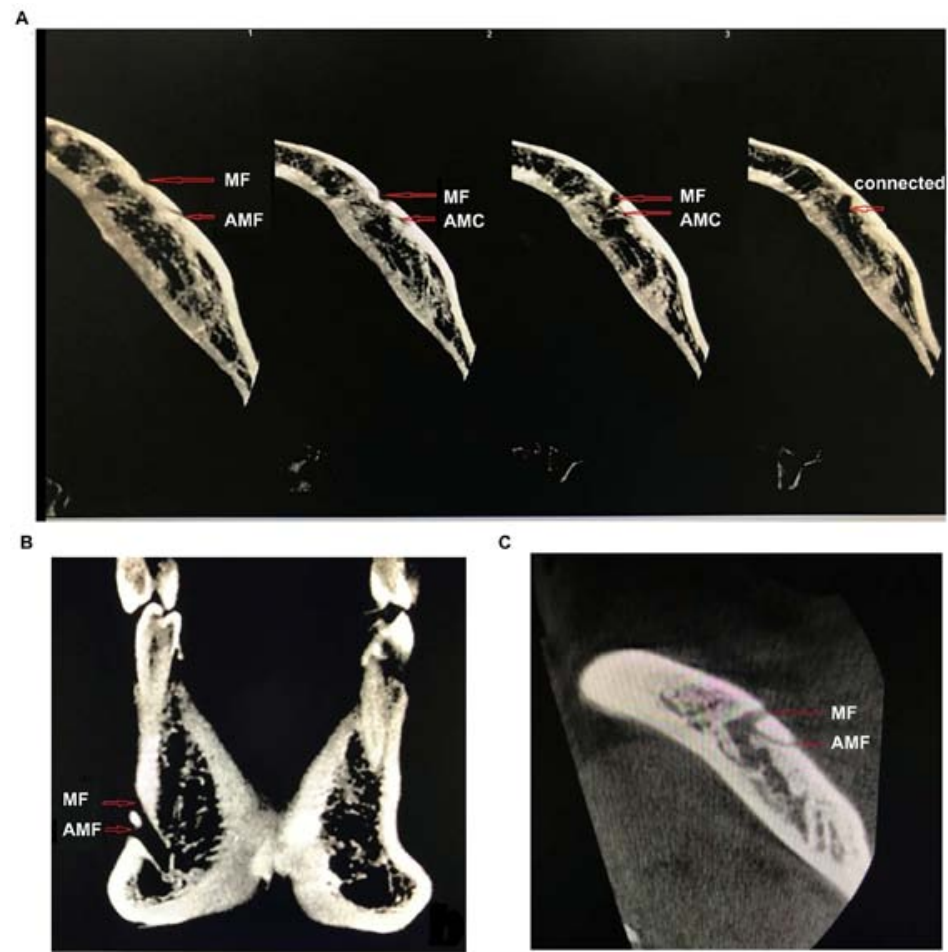

Figure 2. Confirmation of accessary mental foramina and exclusion of nutrient foramina. (A) Arrows mark accessary mental foramina (AMFs), the mental foramen (MF), accessary mental canals (AMCs) and the mental canal (MC). Continuous axial sections show that the AMCs eventually connected to the MC. (B) Connection of the AMCs to the MC on a sagittal section. (C) Connection of the AMCs to the MC on an axial section. 
Table II. Frequencies of accessary mental foramen (AMF) (N/n\%).

\begin{tabular}{lcccccr}
\hline Sex (N) & $\begin{array}{c}\text { Single unilateral } \\
\text { AMF }\end{array}$ & $\begin{array}{c}\text { Two unilateral } \\
\text { AMF }\end{array}$ & $\begin{array}{c}\text { Four unilateral } \\
\text { AMF }\end{array}$ & $\begin{array}{c}\text { Single bilateral } \\
\text { AMF }\end{array}$ & $\begin{array}{c}\text { Double bilateral } \\
\text { AMF }\end{array}$ & Total \\
\hline Male (256) & $15 / 5.86$ & $1 / 0.39$ & 0 & $2 / 0.78$ & 0 & $18 / 7.03$ \\
Female (27) & $13 / 4.8$ & $1 / 0.39$ & $1 / 0.37$ & $2 / 0.74$ & $1 / 0.37$ & $18 / 6.64$ \\
Total (527) & $28 / 5.31$ & $2 / 0.38$ & $1 / 0.19$ & $4 / 0.76$ & $1 / 0.19$ & $36 / 6.83$ \\
\hline
\end{tabular}

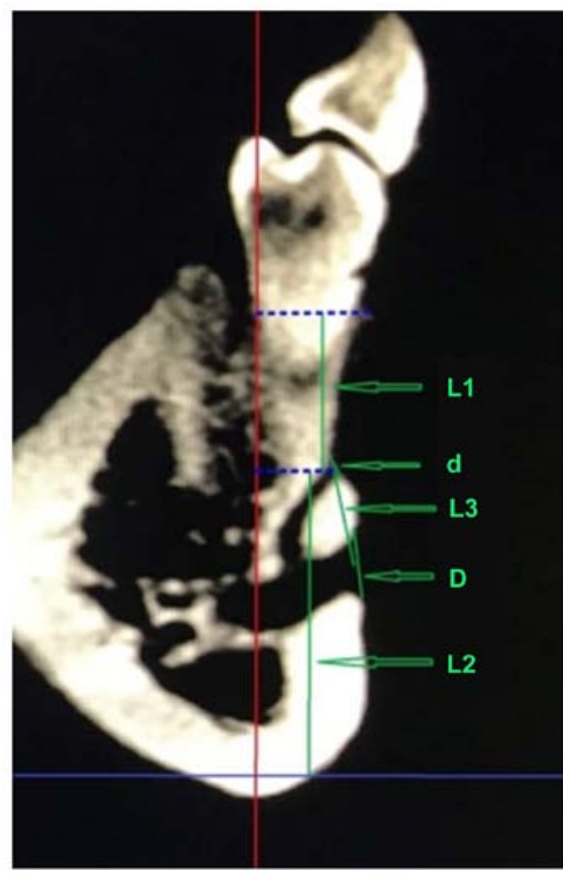

Figure 3. Measuring points as indicated by arrows. d, diameter of the mark accessary mental foramen (AMF); D, diameter of the ipsilateral mental foramen (MF); L1, the distance from the center of the AMF to the alveolar ridge crest; $\mathrm{L} 2$, the distance from the center of the AMF to the mandibular plane; L3, the distance from the center of the AMF to the center of the ipsilateral MF. The blue solid line is the cutting line of the mandibular plane. The red solid line is the cutting line of the sagittal plane. Blue dashed lines are auxiliary lines parallel to the mandibular plane.

with 2 AMFs on each side (Fig. 6). No patient was identified with 3 AMFs unilaterally.

The relative position of AMFs with respect to ipsilateral MFs and adjacent teeth. The relative position of AMFs with respect to ipsilateral MFs is summarized in Table III. The relative position of AMFs with respect to adjacent teeth is summarized in Table IV. In this study, one case of ipsilateral AMFs was observed in an 8-year-old boy with mixed dentition, in which the AMFs were located between the first and second deciduous molars (Fig. 7).

Therefore, AMFs most frequently occurred distal-inferior to the ipsilateral MF, followed by the distal-superior direction. AMFs were most frequently located below the mandibular second premolar, less frequently below the mandibular first molar, and often between the first and second premolars and between the second premolar and the first molar.
Table III. Positions of accessary mental foramen (AMF) relative to mental foramen (MF).

\begin{tabular}{lcc}
\hline Position & Number (n) & Percentage (\%) \\
\hline Mesial-superior & 6 & 12.50 \\
Mesial-inferior & 4 & 8.33 \\
Distal-superior & 11 & 22.91 \\
Distal-inferior & 27 & 56.25 \\
\hline
\end{tabular}

Table IV. Position of accessary mental foramen (AMF) relative to adjacent teeth.

\begin{tabular}{lcc}
\hline Position & Number (n) & Percentage (\%) \\
\hline Below 2nd premolar & 16 & 33.33 \\
Below 1st molar & 12 & 25 \\
$\begin{array}{l}\text { Between 1st and } \\
\text { 2nd premolars }\end{array}$ & 9 & 18.75 \\
$\begin{array}{l}\text { Between 2nd premolar } \\
\text { and 1st molar }\end{array}$ & 9 & 18.75 \\
$\begin{array}{l}\text { Between 1st and 2nd } \\
\text { primary molars }\end{array}$ & 1 & 2.08 \\
Below 1st premolar & 1 & 2.08 \\
\hline
\end{tabular}

The diameters of AMFs and ipsilateral MFs. The center distances, the distances from AMFs to the ARC and to the mandibular plane were statistically analyzed (Table V), and a correlation analysis was performed (Table VI). The average diameter of the 48 AMFs detected was $1.32 \pm 0.61 \mathrm{~mm}$, with $33(68.75 \%)$ larger than $1 \mathrm{~mm}$. The average diameter of the ipsilateral MFs was $3.26 \pm 0.90 \mathrm{~mm}$. There was no correlation between the diameter of the AMFs and that of the ipsilateral MFs. The average center distance between the AMFs and the ipsilateral MFs was $5.42 \pm 2.47 \mathrm{~mm}$, ranging from 1.89 to $13.21 \mathrm{~mm}$, it was not correlated with the diameter of the AMFs and was significantly larger in females than in males $(\mathrm{P}=0.015)$. The average distance from the AMFs to the ARC was $15.05 \pm 3.50 \mathrm{~mm}$ and to the mandibular plane was $15.87 \pm 3.64 \mathrm{~mm}$. There was no correlation between AMF diameter and its average distance to the ARC, to the mandibular plane, or to the average center of the ipsilateral MFs $(\mathrm{P}>0.05)$. Our summary results are presented in Fig. 8 . 

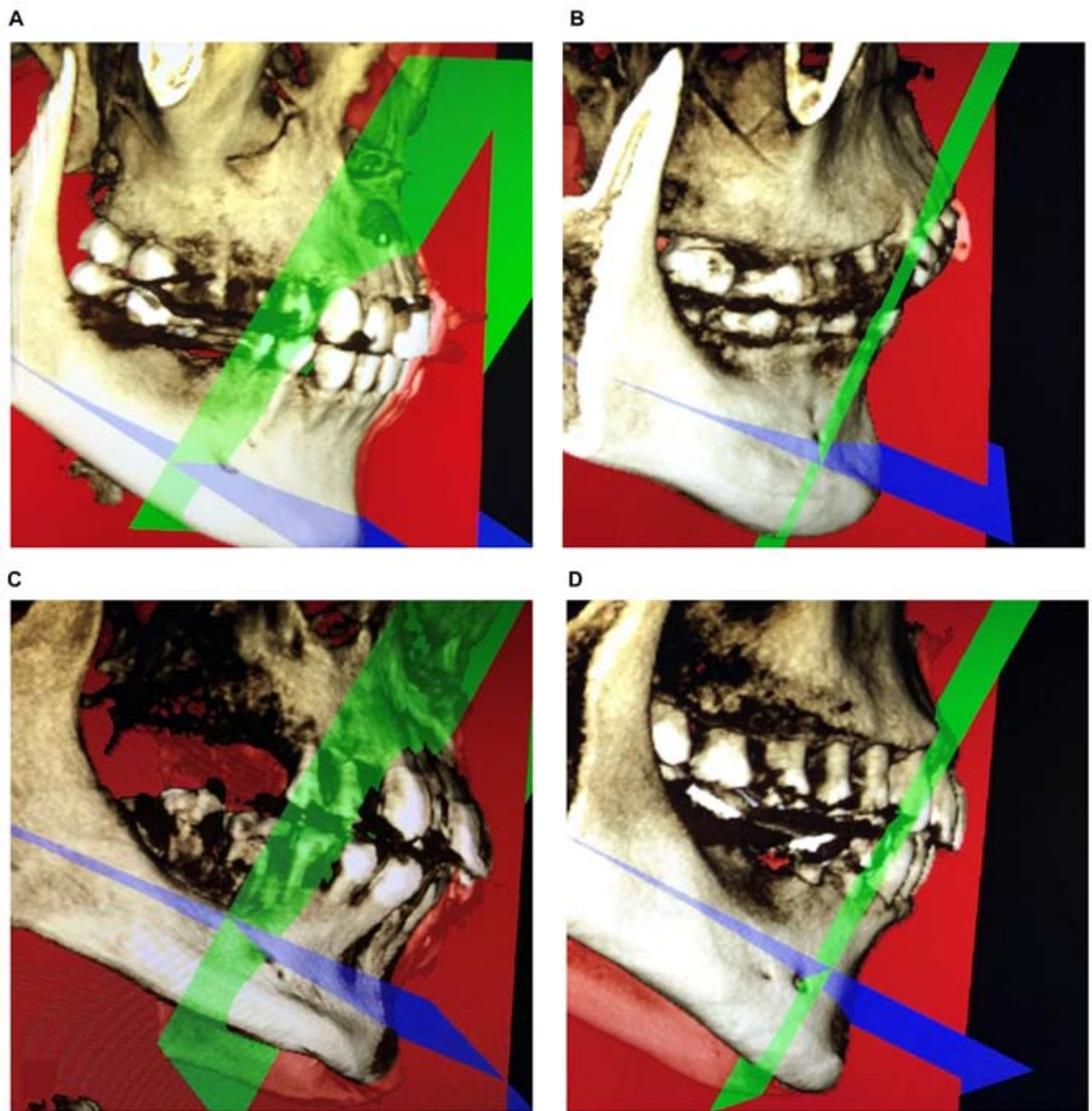

Figure 4. The position of the mark accessary mental foramen (AMF) was described as (A) mesial-superior, (B) distal-superior, (C) distal-inferior and (D) distal-inferior to the mental foramen (MF).
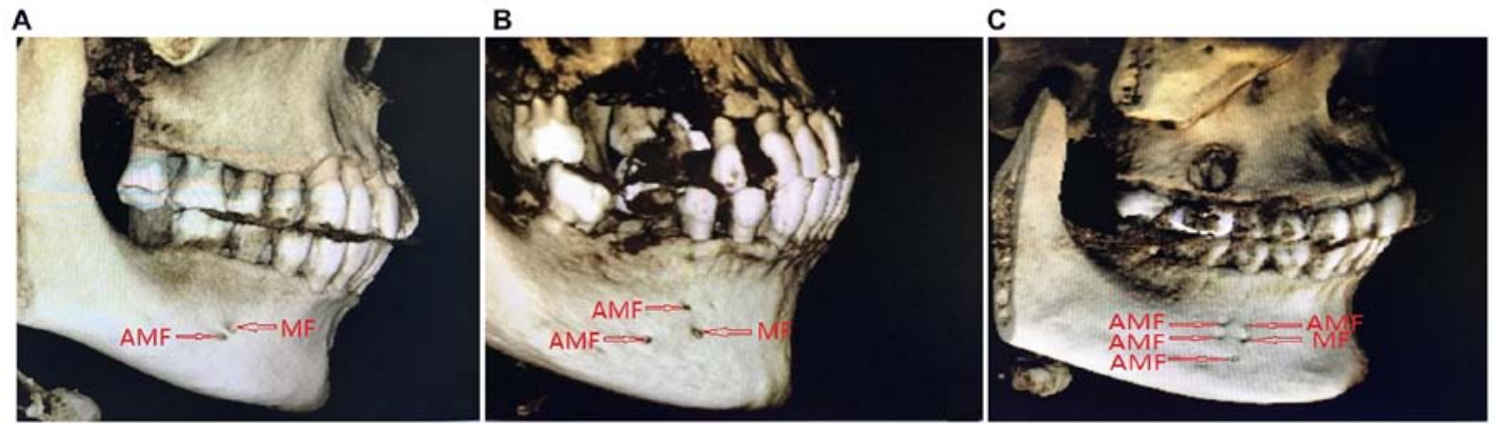

Figure 5.3D images of a unilateral mark accessary mental foramina (AMFs). (A) A single unilateral AMF. (B) Two unilateral AMFs. (C) Four unilateral AMFs.
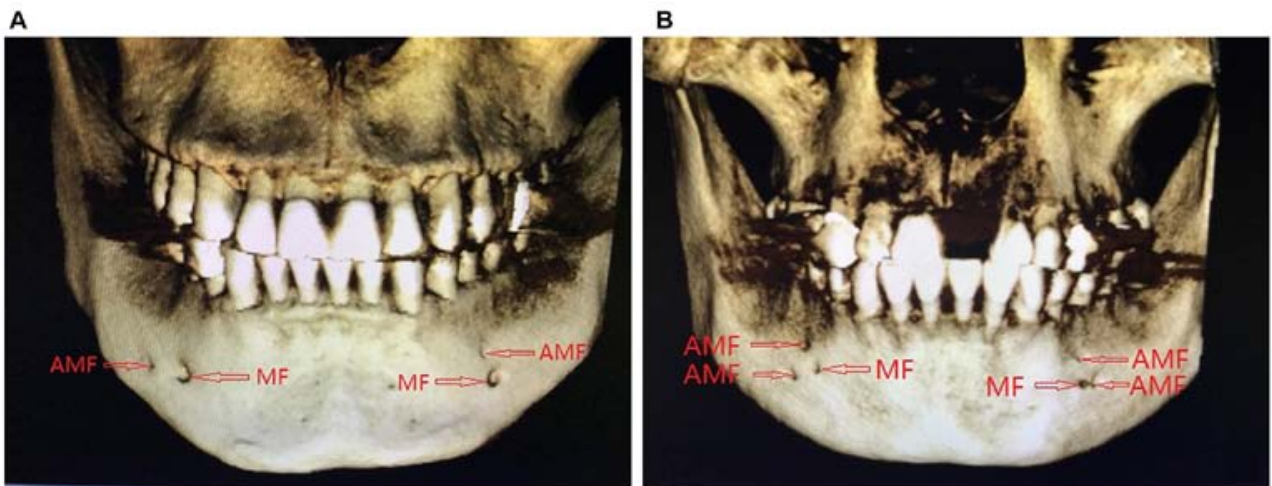

Figure 6.3D images of bilateral accessary mental foramina (AMFs). (A) A single pair of bilateral AMFs. (B) Two pairs of bilateral AMFs. 
Table V. Grouped comparison analysis (mean $\pm \mathrm{SD}, \mathrm{mm}$ ).

\begin{tabular}{lrrrrrrrr}
\hline & \multicolumn{3}{c}{ Left vs. right } & & \multicolumn{3}{c}{ Sex } \\
\cline { 2 - 3 } Group & \multicolumn{1}{c}{ Left (25) } & Right (23) & P value & & Male (21) & Female (27) & P value & Mean \pm SD \\
\hline d & $1.30 \pm 0.60$ & $1.35 \pm 0.64$ & 0.868 & & $1.27 \pm 0.63$ & $1.36 \pm 0.60$ & 0.916 & $1.32 \pm 0.61$ \\
D & $3.52 \pm 0.97$ & $2.97 \pm 0.75$ & 0.365 & & $3.32 \pm 0.99$ & $3.21 \pm 0.85$ & 0.444 & $3.26 \pm 0.90$ \\
L1 & $14.53 \pm 3.81$ & $15.62 \pm 3.12$ & 0.324 & & $14.99 \pm 3.78$ & $15.10 \pm 3.34$ & 0.797 & $15.05 \pm 3.50$ \\
L2 & $16.12 \pm 3.55$ & $15.52 \pm 3.78$ & 0.810 & & $15.84 \pm 4.05$ & $15.90 \pm 3.37$ & 0.260 & $15.87 \pm 3.64$ \\
L3 & $5.96 \pm 2.89$ & $4.84 \pm 1.08$ & 0.071 & & $4.75 \pm 1.50$ & $5.95 \pm 2.95$ & 0.015 & $5.42 \pm 2.47$ \\
\hline
\end{tabular}

$\mathrm{d}$, diameter of accessary mental foramen (AMF); D, diameter of ipsilateral mental foramen (MF); L1, the distance from the center of AMF to alveolar ridge crest; L2, the distance from the center of AMF to mandibular plane; L3, distance from the center of AMF to the center of ipsilateral MF. $\mathrm{P}<0.05$ indicates statistically significant difference.

Table VI. Correlation analysis between diameter of accessary mental foramen (AMF) or mental foramen (MF) and each distance.

Correlation

Distance from the center of AMF to alveolar ridge crest (L1)

Distance from the center of AMF to mandibular plane (L2)

Distance from the center of AMF to the center of ipsilateral MF (L3)
Diameter of AMF (d)

Diameter of MF (D)

$\mathrm{P}<0.05$ indicates statistically significant difference.

A

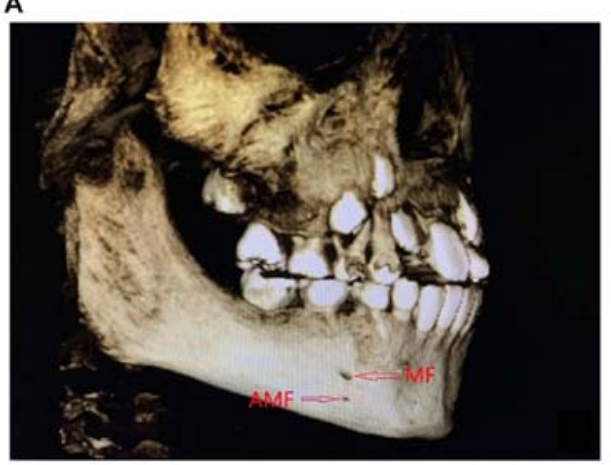

B

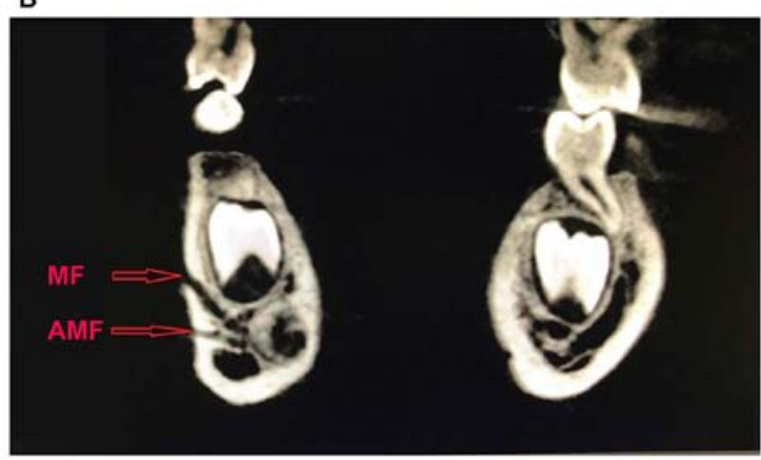

Figure 7. Unilateral mark accessary mental foramen (AMF) with mixed dentition. (A) 3D reconstruction image. (B) Sagittal plane.

\section{Discussion}

The physiological anatomy and clinical significance of $M F$ and $A M F$. The mandibular nerve canal is a complex pipe network that, in most cases, opens at the MFs as a major route. AMFs are anatomic variation of MFs (3) that contain nerves and blood vessels. AMFs have important significance in surgical procedures, including oral implants, apical surgery, orthognathic surgery and tumor resection. In recent years, many studies on AMFs have suggested that the existence of AMFs may lead to surgical complications such as incomplete anesthesia, intraoperative hemorrhage and inferior alveolar nerve injury (4-6). Boronat López and Peñarrocha Diago suggested that the presence of AMFs might be one of the causes of local anesthesia failure, which occurred in $10-20 \%$ of block anesthesia of the mental nerve in patients with accessory mental nerves (7). In addition, damage of the neurovascular bundle transmitted through AMFs during surgery may lead to intraoperative bleeding and postoperative numbness of the lower lip and chin (3). Therefore, it is of great significance for clinical practice to become familiarized with the MFs and their anatomical variation.

Current research status of AMFs

Observation method for AMFs. The most accurate and direct observation of the anatomical structure of the MF region was made on human skull specimens. Early studies of AMFs were carried out through skull specimen observation (8). However, the availability of skull specimens is very limited, and it is difficult to acquire sufficiently large amounts of observation 


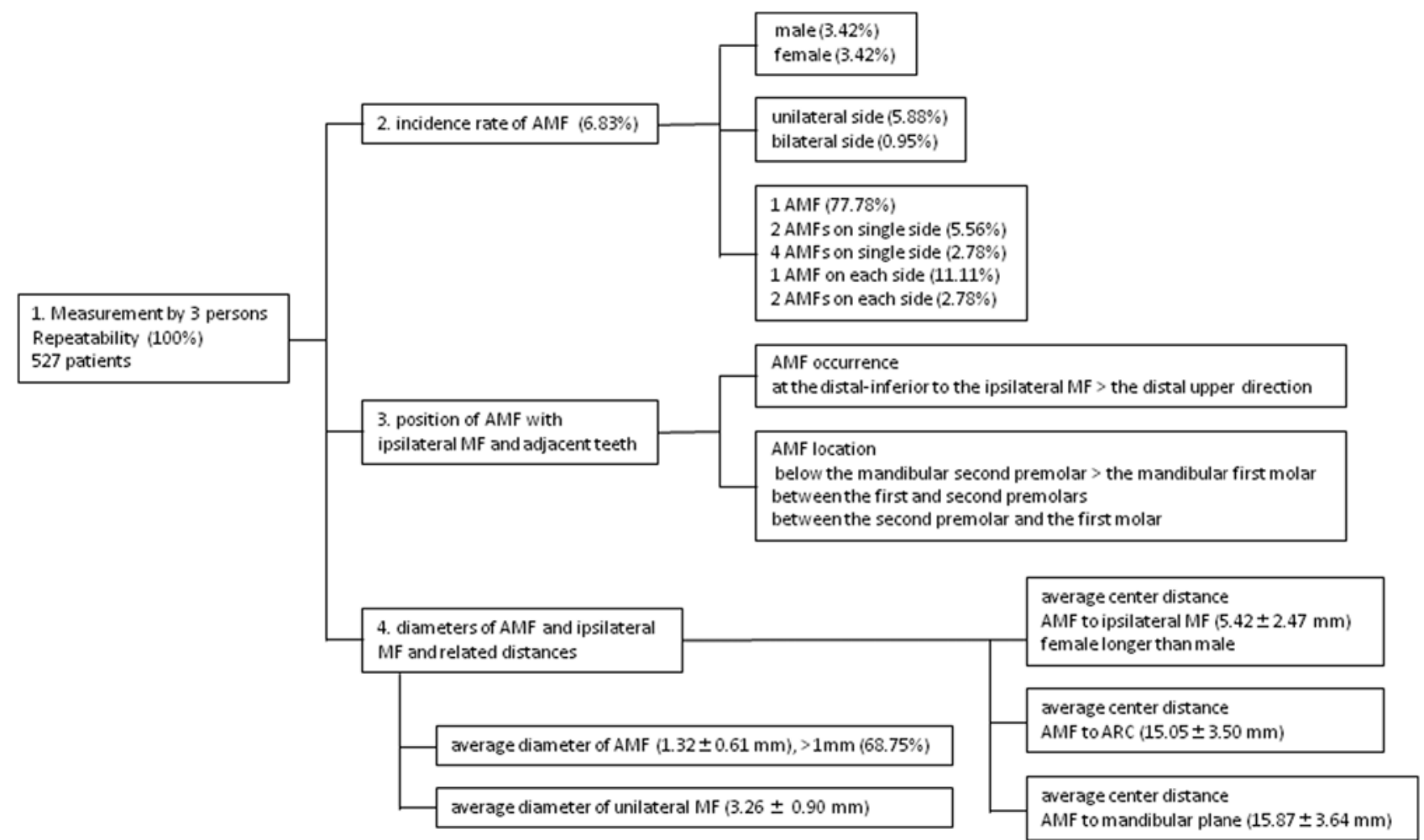

Figure 8 . Flow chart of the summary results.

data to instruct clinical practice. Therefore, imaging techniques are typically used by researchers. As the most commonly used imaging examination method, intraoral apex film and panoramic film benefit from low costs but cannot accurately reflect the anatomical structure of the oral cavity due to overlaps and distortions. AMFs could be found only in individual case reports (9). Spiral CT and CBCT are 3D imaging techniques that can accurately reflect the anatomical structures of the mandible and their positional relationships. Studies have suggested that CBCT has a higher accuracy in the observational analysis of the MFs and the mandibular nerve anterior ring (10-13). The study by Imada et al on the CBCT data and panoramic radiographs of 100 patients suggested an identification rate of $3 \%$ for AMFs by CBCT, while no AMFs were observed on panoramic radiographs (14). In addition, spiral CT suffers from high amounts of radiation, complicated operation and high economic costs, while CBCT is popular among dentists due to its high resolution and clear images, the low amount of radiation it produces and the ability to accurately and clearly reflect the anatomical structure of the oral cavity. CBCT has now become an important method for diagnosing AMFs. Therefore, in this study, CBCT was chosen for the measurement and study of AMFs.

Analysis of the incidence of AMFs and related influencing factors. To date, there are no unified diagnostic criteria for AMFs. Pancer et al proposed that AMFs must be distinguished from the mandibular canal orifice (15). We believe that only those foramina whose lumens are connected to the mental tube and that open on the surface of the mandible can be defined as AMFs. Therefore, this study adopted strict inclusion criteria to ensure the high credibility of the data. The incidence of AMFs is inconsistent in worldwide reports $(0.88-10.66 \%)(3,8,16,17)$. The study by Sawyer et al analyzed human skulls and suggested that the incidence of AMFs was not the same among the populations of different countries and regions, which was considered to be related to ethnicity (17). The incidence of AMFs is $1.4 \%$ in white Americans, $5.7 \%$ in African Americans, $1.5 \%$ in Asian Indians and $9.0 \%$ in Pre-Columbian Nazca Indians (17). Among the 527 patients in this study, the incidence of AMFs was $6.83 \%$, which is lower than that of Columbian Indians but higher than that of white Americans, African Americans and Asian Indians. Hanihara and Ishida studied 81 human populations from around the world and found that the frequency of AMFs was the highest in middle Asian and Subsaharan African populations, followed by European, South Asian, East Asian, Southeast Asian, Western Australia and South American populations (18). This study focused on the Chinese Han population, and strict inclusion and exclusion criteria were adopted, which excluded buccal foramina of the branch of the mandibular nerve canal or mandibular incisor canal and also rolled out nutrient foramina formed by the branches of the facial artery and the submental artery transmitting through the mandible. Our results were true and accurate and provide an anatomical basis for the guidance of clinical operations. Naitoh et al reported that in 157 patients, 11 had AMFs (7\%), in which 2 cases had bilateral AMFs (19). Katakami et al investigated the CBCT data from 170 patients and found 16 cases with AMFs (10.7\%), which only included 1 case of bilateral AMFs (3). Oliveira-Santos et al studied the CBCT data from 285 patients and identified 27 cases with AMFs (9.4\%), including 2 cases of bilateral AMFs (20). Haktanir et al examined 100 patients with multislice spiral CT and found 4\% with AMFs (21), while Imada et al used 
CBCT to study the same number of patients and found that the frequency of AMFs was 3\%, including 2 cases of unilateral AMFs and 1 case of bilateral AMFs (14). In summary, multiple studies have suggested that the incidence of AMFs is $~ 4-10.7 \%$. The proportion of patients with bilateral AMFs is very small, accounting for $7.41 \%$ of all patients with AMFs (20). Our study investigated 527 patients and found that the frequency of AMF was $6.83 \%$, including 5 cases of bilateral AMFs, which constituted $13.89 \%$ of all AMFs and $0.95 \%$ of all cases. Our result was not very consistent with the above studies, which might be explained by differences in ethnicity, diagnostic standards, research methods and sample sizes. In addition, the layer thickness could reach $0.2 \mathrm{~mm}$ when using CBCT to observe AMFs, while some other studies used traditional spiral CT, which may have affected the results.

Sex differences in AMFs. Our study found no difference in the frequency of AMFs between sexes, which is consistent with most studies worldwide $(9,16,19)$. However, the study by Hanihara and Ishida on skulls suggested that the frequency of AMFs is highest in Asian males (18). Some studies have reported that the incidence of AMFs is higher in females than in males (22), while other studies suggest otherwise $(17,20)$. This variance in AMF frequency between sexes is possibly due to the different patient ethnicities, sample sizes and research methods.

Relative positions of AMFs with respect to ipsilateral MFs and adjacent teeth. Internationally, there is no unified standard for describing the relative location of AMFs with respect to ipsilateral MFs. Our study used the tangent of the axial plane, which was parallel to the mandibular plane and passed through the MFs, on the buccal cortical surface of the mandible as the horizontal axis and the tangent of the coronal plane, which was vertical to the mandibular plane and passed through the MFs, on the buccal cortical surface of the mandible as the vertical axis to accurately divide the MF region into four quadrants for describing the relative location of AMF. We believe this partition is clear and accurate and provides a standard positioning system for surgery, which can facilitate data comparison. Naitoh et al found that in the Japanese population, AMFs were mostly located distal-inferior with respect to the MFs, and the distance between the MFs and AMFs was $6.3 \mathrm{~mm}$ on average (19). Katakami et al observed that 10 out of 17 AMFs $(59 \%)$ were positioned in the posterior and $8(47 \%)$ in the inferior area relative to the MFs (3). In this study, AMFs were most frequently positioned distal-inferior to the ipsilateral MF, accounting for $56.25 \%$ of all AMFs, followed by the distal-superior position, which accounted for $22.91 \%$ of all AMFs. This result is consistent with previous reports, but our partitioning of the MF region could describe the positional relationship between AMFs and the MFs more standardly and accurately, which can be beneficial for repeated comparisons worldwide. The research by Kalender et al found that in the Turkish population, AMFs were mostly positioned in an anteroinferior position with respect to the MFs (16). Imada et al suggested that most AMFs were located between the premolars, either superiorly or mesially to the MFs (14). These two reports are in disagreement with our result, which might be due to the differences in ethnicity and diagnostic criteria.
Based on a study of 150 adult dry human mandibles, Voljevica et al found that $60.30 \%$ of AMFs were located at the root of the mandibular first premolar and $20.3 \%$ were located between the mandibular first and second premolar (23). As reported by Katakami et al, mandibular AMFs were frequently observed inferior to the root apex of the mandibular second premolars (3). In this study, AMFs were mainly located below the mandibular second premolars (33.33\%), which is consistent with Katakami's study, and, less frequently, inferior to the mandibular first premolar, between the first and second premolars and between the second premolar and the first molar, indicating the diversity of the relative positions between AMFs and ipsilateral teeth. In clinical practice, attention should be paid to the presence of AMFs during implant surgery at the $\mathrm{MF}$ region and in chin surgery.

Katakami et al reported that the diameter of the mandibular AMFs was $1.2 \mathrm{~mm}$ (3). Naitoh et al reported an average size of $1.5 \mathrm{~mm}$ for AMFs and suggested that the frequency of AMFs was unrelated to the diameter of the MFs after comparing the sizes of the ipsilateral and contralateral MFs (24). The average diameter of the AMFs measured in this study was $1.32 \mathrm{~mm}$, with $33(68.75 \%)$ larger than $1 \mathrm{~mm}$. The average diameter of the ipsilateral MF was $3.26 \mathrm{~mm}$. There was no correlation between the diameter of the AMFs and ipsilateral MFs, which agreed with the above study. Our results showed that the size of AMFs is evidently smaller than that of the MFs, making them easily distinguished. However, another study (20) reported that in $37 \%$ of the cases, the diameter of AMFs was comparable to or larger than half of the diameter of the MFs and that the AMF/MF diameter ratio could reach as high as 0.99 , suggesting that the sizes of some AMFs were close to that of the MFs.

The study by Naitoh et al suggested that the average distance between AMFs and the ipsilateral MF was $6.3 \mathrm{~mm}$ (19). Our result found an average distance of $5.42 \mathrm{~mm}$ between the AMFs and the MFs, ranging from 1.89 to $13.21 \mathrm{~mm}$, which was unrelated to the diameters of the AMFs. These data were smaller than those in the Naitoh study but larger than those in the study by Sisman et al (3.56 mm) (25). Clinically, $2 \mathrm{~mm}$ above the MF is generally considered the safety zone for implantation. Considering that the average distance of $5.42 \mathrm{~mm}$ between the AMFs and the MFs was far beyond the $2 \mathrm{~mm}$ range, we suggest that this safety distance is debatable, which is in agreement with the opinion of Al-Mahalawy et al (26). The safety distance should be set in consideration of the presence of AMFs. The difference between our data and previous results can likely be ascribed to the different imaging techniques, inclusion criteria or other factors. As this study enrolled mainly individuals from the Chinese Han population within Shandong Province, the results of our survey might only apply to the population in the survey area, and a larger sample size and survey region may be required for future study.

Distance from the AMFs to the ARC and mandibular plane. The ARC is the highest point of the alveolar bone and is visible in the mouth. Measuring the distance between the AMFs and the ARC could indirectly facilitate the determination of the position of the MFs and AMFs for avoiding damage to blood vessels and nerves. The mandibular plane is a standard plane 
commonly used in stomatology. In this study, we used the mandibular plane rather than the mandibular margin, which is often used in China, as the measurement plane, which was beneficial for the repeatability of the experiment and for making comparisons with international data. Currently, there are few reports of measurements of the distances from the AMFs to the ARC and mandibular plane. This is likely due to the lack of sufficient attention to the anatomical variations in the AMFs. Our results showed that the average distance between the AMFs and the ARC was $15.08 \mathrm{~mm}$ and that between the AMF and the mandibular plane was $15.87 \mathrm{~mm}$. The shortest distance between an AMF and the ARC was $6.80 \mathrm{~mm}$, in which the AMF was larger than $1 \mathrm{~mm}$. Therefore, during an operation, damage should be avoided to prevent hemorrhage or numbness. In addition, in one's lifetime, the alveolar bone is constantly rebuilt and maintains the ability to dynamically change. Our result could not dynamically reflect the distances between the AMFs and the ARC, which was a limitation of this study.

In conclusion, first in regards to the confirmation of AMFs, nutrient foramina around the MFs were excluded. An AMF was defined as an opening of the branch of the mental canal on the outer surface of the mandible. Second, the reference plane for measuring AMFs was suggested to be the mandibular plane to increase the repeatability and accuracy of the experiment. Third in regard to the standard for determining the relative positions of the AMFs and the MFs, the tangent of the axial plane, which was parallel to the mandibular plane and passed through the MFs, on the buccal cortical surface of the mandible was used as the horizontal axis. The tangent of the coronal plane, which was vertical to the mandibular plane and passed through the MFs, on the buccal cortical surface of the mandible was used as the vertical axis. The two axes accurately divided the MF region into four quadrants. This internationally unified standard was beneficial to ensuring high compatibility among the data.

Finally, based on our results, we propose that for implant surgeries, the safety region of $2 \mathrm{~mm}$ above the MFs should be reevaluated. If CBCT shows that an AMF is located outside the safety range of $2 \mathrm{~mm}$ from the ipsilateral $\mathrm{MF}$ and that its diameter is larger than $1 \mathrm{~mm}$, indicating that the neurovascular bundle through the AMF is relatively large, this area should be avoided during operation to prevent excessive bleeding or numbness caused by surgical trauma. On the other hand, an AMF smaller than $1 \mathrm{~mm}$ as seen on CBCT indicates a smaller neurovascular bundle through the AMF. In such cases, surgical trauma is unlikely to cause serious consequences. Therefore, a standard safe distance of $2 \mathrm{~mm}$ can still be applied. CBCT examination is recommended before the operation to identify important anatomical structures around the MF region and their variations and to set the safety region on an individual basis.

\section{Acknowledgements}

Not applicable.

\section{Funding}

No funding was received.

\section{Availability of data and materials}

The datasets used and/or analyzed during the present study are available from the corresponding author on reasonable request.

\section{Authors' contributions}

LX wrote the manuscript. LX and WP were responsible for establishing the reference plane and performing the data measurement. HB and XH contributed to the observation index analysis. The final version was read and adopted by all the authors. All authors read and approved the final manuscript.

\section{Ethics approval and consent to participate}

The study was approved by the Ethics Committee of The Affiliated Yantai Yuhuangding Hospital of Qingdao University (Yantai, China). Patients who participated in this study had complete clinical data. Signed informed consent was obtained from the patients and/or guardians for the publication of any associated data and accompanying images.

\section{Patient consent for publication}

Not applicable.

\section{Competing interests}

The authors declare that they have no competing interests.

\section{References}

1. Koutroumpas DC and Koletsi-Kounari H: Galen on dental anatomy and physiology. J Hist Dent 60: 37-49, 2012.

2. da Silva Ramos Fernandes LM, Capelozza AL and Rubira-Bullen IR: Absence and hypoplasia of the mental foramen detected in CBCT images: A case report. Surg Radiol Anat 33: 731-734, 2011.

3. Katakami K, Mishima A, Shiozaki K, Shimoda S, Hamada Y and Kobayashi K: Characteristics of accessory mental foramina observed on limited cone-beam computed tomography images. J Endod 34: 1441-1445, 2008.

4. Muinelo-Lorenzo J, Suárez-Quintanilla JA,Fernández-Alonso A, Varela-Mallou J and Suárez-Cunqueiro MM: Anatomical characteristics and visibility of mental foramen and accessory mental foramen: Panoramic radiography vs. cone beam CT. Med Oral Patol Oral Cir Bucal 20: e707-e714, 2015.

5. Torres MG, Valverde LF, Vidal MT and Crusoé-Rebello IM: Accessory mental foramen: A rare anatomical variation detected by cone-beam computed tomography. Imaging Sci Dent 45: 61-65, 2015

6. Cantekin K and Sekerci A: Evaluation of the accessory mental foramen in a pediatric population using cone-beam computed tomography. J Clin Pediatr Dent 39: 85-89, 2014.

7. Boronat López A and Peñarrocha Diago M: Failure of locoregional anesthesia in dental practice. Review of the literature. Med Oral Patol Oral Cir Bucal 11: E510-E513, 2006.

8. Zografos $\mathbf{J}$ and Mutzuri A: Incidence of double mental foramen in a sample of Greek population. Odontostomatol Proodos 43: 521-523, 1989 (In Greek).

9. Cağirankaya LB and Kansu H: An accessory mental foramen: A case report. J Contemp Dent Pract 9: 98-104, 2008.

10. Kamburoğlu K, Kiliç C, Ozen T and Yüksel SP: Measurements of mandibular canal region obtained by cone-beam computed tomography: A cadaveric study. Oral Surg Oral Med Oral Pathol Oral Radiol Endod 107: e34-e42, 2009.

11. Matherne RP, Angelopoulos C, Kulild JC and Tira D: Use of cone-beam computed tomography to identify root canal systems in vitro. J Endod 34: 87-89, 2008. 
12. Vujanovic-Eskenazi A, Valero-James JM, Sánchez-Garcés MA and Gay-Escoda C: A retrospective radiographic evaluation of the anterior loop of the mental nerve: Comparison between panoramic radiography and cone beam computerized tomography. Med Oral Patol Oral Cir Bucal 20: e239-e245, 2015.

13. Santana RR, Lozada J, Kleinman A, Al-Ardah A, Herford A and Chen JW: Accuracy of cone beam computerized tomography and a three-dimensional stereolithographic model in identifying the anterior loop of the mental nerve: A study on cadavers. J Oral Implantol 38: 668-676, 2012.

14. Imada TS, Fernandes LM, Centurion BS, de Oliveira-Santos C, Honório HM and Rubira-Bullen IR: Accessory mental foramina: Prevalence, position and diameter assessed by cone-beam computed tomography and digital panoramic radiographs. Clin Oral Implants Res 25: e94-e99, 2014.

15. Pancer B, Garaicoa-Pazmiño C and Bashutski JD: Accessory mandibular foramen during dental implant placement: Case report and review of literature. Implant Dent 23: 116-124, 2014.

16. Kalender A, Orhan K and Aksoy U: Evaluation of the mental foramen and accessory mental foramen in Turkish patients using cone-beam computed tomography images reconstructed from a volumetric rendering program. Clin Anat 25: 584-592, 2012.

17. Sawyer DR, Kiely ML and Pyle MA: The frequency of accessory mental foramina in four ethnic groups. Arch Oral Biol 43 . $417-420,1998$

18. Hanihara $\mathrm{T}$ and Ishida $\mathrm{H}$ : Frequency variations of discrete cranial traits in major human populations. IV. Vessel and nerve related variations. J Anat 199: 273-287, 2001.

19. Naitoh M, Hiraiwa Y, Aimiya H, Gotoh K and Ariji E: Accessory mental foramen assessment using cone-beam computed tomography. Oral Surg Oral Med Oral Pathol Oral Radiol Endod 107: 289-294, 2009.
20. Oliveira-Santos C, Souza PH, De Azambuja Berti-Couto S, Stinkens L, Moyaert K, Van Assche $\mathrm{N}$ and Jacobs R: Characterisation of additional mental foramina through cone beam computed tomography. J Oral Rehabil 38: 595-600, 2011.

21. Haktanir A, Ilgaz K and Turhan-Haktanir N: Evaluation of mental foramina in adult living crania with MDCT. Surg Radiol Anat 32: 351-356, 2010.

22. Kieser J, Kuzmanovic D, Payne A, Dennison J and Herbison P Patterns of emergence of the human mental nerve. Arch Oral Biol 47: 743-747, 2002.

23. Voljevica A, Talović E and Hasanović A: Morphological and morphometric analysis of the shape, position, number and size of mental foramen on human mandibles. Acta Med Acad 44: 31-38, 2015.

24. Naitoh M, Yoshida K, Nakahara K, Gotoh K and Ariji E: Demonstration of the accessory mental foramen using rotational panoramic radiography compared with cone-beam computed tomography. Clin Oral Implants Res 22: 1415-1419, 2011.

25. Sisman Y, Sahman H, Sekerci A, Tokmak TT, Aksu Y and E: Detection and characterization of the mandibular accessory buccal foramen using CT. Dentomaxillofac Radiol 41: 558-563, 2012.

26. Al-Mahalawy H, Al-Aithan H, Al-Kari B, Al-Jandan B and Shujaat S: Determination of the position of mental foramen and frequency of anterior loop in Saudi population. A retrospective CBCT study. Saudi Dent J 29: 29-35, 2017. International (CC BY-NC-ND 4.0) License. 